\title{
Ein Plädoyer für die epistemologische Semantik. Einige Überlegungen zu ihrer Konzeption, zu ihren Aufgaben und zur Analyse der polnischen Fachtextäußerungen im Fachgebiet ,altgriechische Musik'1
}

\author{
Towards clarity on epistemological semantics. \\ Some thoughts on its theory and tasks based \\ on an analysis of Polish specialized expressions \\ in the music of ancient Greeks
}

\begin{abstract}
By way of this article the author seeks to construe epistemological semantics. To this end the author presents his arguments in several aspects as regards a) concepts, b) terms, c) subject matter, d) methods and e) analysis of expressions excerpted from texts. The author's concept is based on hypotheses employed in anthropocentric linguistics and frame semantics. One of the building blocks of epistemological semantics is constituted by so called epistemic formants of knowledge, understood as the foundation of epistemic experience, as the base for the frames of epistemic knowledge, and hence also as the framework for the constitution of meanings and expressions formed by an individual. As important seem a strict delimitation of the subject area of epistemological semantics and the defining of resultant tasks to be undertaken by linguists. It transpires that both the subject area and the resultant tasks have up till now been restricted almost exclusively to 'meaning', without clear reference to the underlying cognitive acts. Methods of the work are rooted in idiocentric (individual) and polycentric (common) approaches known in lin-
\end{abstract}

${ }^{1}$ Dieser Beitrag versteht sich als Fortsetzung des in Glottodidactica 1/2015 erschienen Textes Kognitiv und/oder epistemisch. Auf dem Weg zur epistemologischen Semantik. 
guistics. The results of the empirical analysis have been presented and exemplified by a selected specialised neologism from the field of music of Ancient Greece.

KEYWORDS: epistemic; specialist semantics; epistemic formants of knowledge; creation of meanings and specialised expressions.

\section{PROBLEMSTELLUNG}

Dass sich die Semantik als eine linguistische Teildisziplin versteht, im Rahmen derer einzelne Ausdrücke bis hin zu Texten und Diskursen erfasst werden, ist unbestreitbar. Unmissverständlich bleibt dabei die Tatsache, welch komplexe und damit schwierige Herausforderung es darstellt, Textäußerungen, darunter Fachtextäußerungen auf alle Nuancen der Äußerungsform zu examinieren und diese als motivierte Regel zu Papier zu bringen. Noch komplexer und noch schwieriger ist es, anhand von Textäußerungen etwas über die mentalen Fakten (sog. Repräsentationen) ihrer Träger, d.h. der menschlichen Subjekte auszusagen, die diese mentalen Fakten invozieren und/oder evozieren (vgl. Fillmore 1982 zit. nach Busse 2012: 122-131). Wie sich einzelne Forscher dabei positionieren, und zwar von welcher semantischen Schule sie konzeptuell ausgehen, wie sie ihre Grundannahmen verorten und welche Forschungsmethoden sie anwenden, hängt nicht nur davon ab, was sie an semantisch profilierten Konzeptionen oder Theorien letztendlich überzeugt, sondern, wie angemessen und wissenschaftlich reliabel sich damit konkrete Textäußerungen beschreiben lassen. Unabhängig davon, wie weit manche Konzeptionen voneinander entfernt sind, z. B. die der mentalistischen und gebrauchstheoretischen Schule, so ist ihnen eins gemeinsam: der eigentliche Gegenstand und die darauf zu beziehenden Forschungsbereiche und -interessen der Semantik bleiben recht verschwommen. Die Vertreter der Semantikschulen sind sich nämlich darin nicht einig, was untersucht werden soll: (a) die Ausdrucksform einer Äußerung, (b) die „Bedeutung einer Äußerung“, (c) die polykulturellen und/oder polylektalen Faktoren, die sie determinieren, (d) die Funktionen, die einer Äußerungsform zugeschrieben werden oder (e) die kognitiven Prozesse, die diese Funktionen steuern und voraussetzen? Stellt abermals die sanktionierte Äußerungsform das entscheidende Kriterium dar, wenn konkrete Textäußerungen auf die semantische Wirklichkeit ihrer Träger, und genauer, auf die wirklich aktivierten Wissenselemente in einem sprachlichen und kulturellen Handlungsakt, examiniert werden? (vgl. Pawłowski 2012: 253-256).

Abseits der semantisch orientierten Reflexion und der empirischen Analyse sind die epistemische Erfahrung und die epistemischen Prozesse zu 
nennen, die die kognitiven Fakten überhaupt präsupponieren und damit die Konstitution der Idiokultur möglich machen (näher zur Idiokultur s. F. Grucza 1988, 1992, 2000). Erwähnt sei außerdem die Fähigkeit, die Idiokultur durch sprachliche und/oder kulturelle Äußerungen, also gezielte Handlungsmittel, zu kommunizieren. Genügt es in diesem Lichte nach der „Bedeutung eines Ausdrucks" allein oder nach seiner "pragmatischen Funktion“ zu fragen? Vielmehr sollte man der epistemischen Fähigkeit und den Erkenntnisakten auf den Grund gehen, die der idiolektalen Ausdrucks- und Bedeutungskonstitution und final der Konstruktion einer jeden sprachlichen/kulturellen Äußerung vorausgehen. Damit will gesagt werden, dass die idiolektale Ausdrucks- und Bedeutungskonstitution jeweils neu und prinzipiell epistemisch geformt wird, und das nicht nur bei Wortneubildungen.

Um herauszufinden, warum dem so ist, soll sich die Semantik so positionieren, damit die (a) epistemische Fähigkeit, (b) die epistemische Erfahrung und (c) die (epistemischen) Erkenntnisakte ins Zentrum der Reflexion gerückt werden. Die Erkenntnisakte zu erkennen, konzeptuell und methodisch festzuhalten, heißt erläutern und exemplarisch belegen zu können, warum sich zum Beispiel die Ausdrücke ,Morgenstern' und ,Abendstern' nicht wie behauptet wird - durch ihre Konnotationen unterscheiden (polemisch dazu vgl. Pawłowski 2013b: 292-299). Es heißt außerdem, der Frage nachzugehen, wie die Konstitution von Wortneubildungen, zum Beispiel die eines

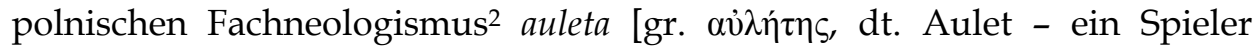
eines altgriechischen Blasinstrumenten aulos, gr. av̉ ós], motiviert ist, oder sogar über die Gründe zu erfahren, warum sich der Übersetzer und zugleich der Innovator dieses Fachneologismus mit Blick auf den musikologischen Fachdiskurs in Polen schließlich für die Äußerungsform auleta und nicht etwa für aulecista oder auletnik entschieden hat (vgl. u. a. Landels 2003: 44, West 2003: 104).

Um zu dokumentieren, dass dies mit dem epistemologisch profilierten Ansatz zur Semantik möglich ist, bemühe ich im diesem Beitrag den Forschungsgegenstand und die daraus resultierenden Forschungsaspekte und aufgaben der epistemologischen Semantik zu besprechen. Dabei nenne ich die epistemischen Wissensformantentypen, d.h. die für die (epistemischen) Erkenntnisakte konstitutive ,Ausstattung' menschlicher Individuen (näher dazu vgl. Pawłowski 2012, 2014, 2015b) und komme anschließend auf die idiolektale Bedeutungs- und Ausdruckskonstitution zu sprechen, um sich schließlich der exemplarischen Darstellung der Forschungsergebnisse $\mathrm{zu}$ zuwenden, die aus meiner Studie zu den polnischen Fachneologismen resultieren.

2 Zur Deutung und Analyse von Fachneologismen siehe Pawłowski 2015b. 


\section{ZUM GEGENSTAND UND ZU DEN AUFGABEN DER EPISTEMOLOGISCHEN SEMANTIK}

Zum Gegenstand der Semantik haben sich mancherlei Linguisten geäußert (vgl. u.a. Katz / Foder 1964; Geckeler 1971; Abraham / Binnich 1972; Engelkamp 1973; Beeh 1973; Lyons 1977; Reis 1980; Birwisch 1983; Lutzeier 1985; Polenz, P. v. 1988; Bock 1990; Schwarz 1992; Dietze 1994; Grabowski et.al. 1996; Grzegorczykowa 2001; Tokarski / Pajdzińska 2001; Bärenfänger 2002). Die Perspektive, aus der dieses Problem in der letzten Dekade beleuchtet wurde, ist jeweils unterschiedlich. Einerseits kreist der Gegenstand um die Bedeutung und wird somit auf Designate, Referenten, Konzepte etc. eingeschränkt, andererseits wird er wortlos übergangen (vgl. u.a. Löbach 2000: 9-11, Metzeltin 2007, u.a. 23, Busse 2012; Ziem 2008; Wunder 2008; Helbig 2008; Schemann 2002; Löbner 2003: 3-11; Wierzbicka 2007, 2008; Linz 2002, Loppe 2010; Tokarski 2004; Kleiber 2003; Mangasser-Wahl 2000; Apresjan 2000, meist überzeugend v.a. Schwarz 1992: 27, näher dazu vgl. Pawłowski 2012: 250-253). Ist das Problem bereits gelöst oder stehen die Semantiker im Kreis ungelöster Rätsel, die einer ständigen Auseinandersetzung bedürfen?

Die Sache ist alles andere als anspruchslos. Hoffnung bringt der epistemologische Ansatz zur Semantik mit sich, dank dem neue Forschungsaspekte und- ziele formuliert werden (vgl. Busse 2007b: 9, 2008a: 70-76, 2008d: 5-11, Pawłowski 2011, 2013a, 2014, 2015b). Der epistemologische Ansatz lässt Insuffizienzen in der Thesenformulierung und in der Methodik der bisherigen Semantikforschung aufkommen (s. Busse 2012: u. a. 12-18) und das Bedürfnis entstehen, alte Voraussetzungen und Forschungsmethoden ergänzen oder sogar verlassen zu müssen. Mit Rückblick auf das seit dem Anfang der 1980er Jahre wachsende Interesse an dem wirklichen Gegenstand und den Aufgaben der angewandten (vgl. F. Grucza v.a. 1983: 274-312) und der anthropozentrischen Linguistik und Kulturologie (vgl. v.a. F. Grucza 2012b), Bonacchi 2009, 2010, 2011a/b, S. Grucza 2010) entwickeln sich im Warschauer Kreis der anthropozentrischen Linguistik Initiativen, die darauf abzielen, die Kultur und die Sprache eines Individuums sowie die Relationen zwischen ihren (wirklichen) Designaten zu verstehen und zu ,beschreiben'. Das Interesse daran wächst in den letzten Jahren vor allem mitsamt der Reflexion um die menschliche Erkenntnisfähigkeit und -arbeit sowie die daraus (zu) folgenden Konsequenzen für die (epistemologische) Linguistik (vgl. S. Grucza 2008: 80-118, 2012: 71-108, F. Grucza 2012b: 294-304). Postulate zur adäquaten Konzeptualisierung und zur linguistischen Anerkennung der menschlichen Erkenntnis werden aufgestellt. 
Die Hervorhebung der Erkenntnisaktivität im Sinne einer besonderen Kategorie von Aktivitäten aller Lebewesen impliziert quasi automatisch die Annahme, dass alle Lebewesen über Fähigkeiten verfügen, bestimmte Erkenntnisaktivitäten auszuführen, dass alle Lebewesen genetisch mit Fähigkeiten ausgestattet sind, die sie in die Lage versetzen, Erkenntnisaktivitäten $\mathrm{zu}$ vollziehen. (F. Grucza 2012b: 295, siehe auch 297).

Parallel dazu kommt im Düsseldorfer Kreis um Dietrich Busse die Idee auf, die linguistische Epistemologie gegen die „üblichen Auffassungen“ der Linguisten jener Zeit zu emanzipieren (Busse 2012: 270, vgl. auch Busse 2005, 2008a/b/c, 2009, 2012: u.a. 11 f., 214, 270, 441, 811, und v.a. 787-818).

Eine differenzierte, dem verstehensrelevanten Wissen in seiner ganzen Breite und Vielfalt gerecht werdende linguistische Semantik kann nach dieser, explizit in den Mittelpunkt gestellten Überlegung nur im Kontext einer noch zu entwickelnden ,linguistischen Epistemologie' (...) erfolgen, die notwendig auf dem Frame-Konzept fußen muss. (Busse 2012: 526).

Busses linguistische Epistemologie versteht sich als Wissenschaft, die darauf abzielt, die Wissenskonstitution im epistemischen Wissensrahmen zu erfassen und zu ,beschreiben'. Schlüssig sind dabei die sog. ,verstehensrelevanten Wissensstrukturen' sowie die innere und äußere (Wissens-)Kontextualierung (ders. 2007a).

Zielobjekt einer linguistischen Epistemologie (oder epistemologischen bzw. verstehensrelevanten Semantik) sind also zunächst und vor allem die mit dem Modell der Frames beschreibbaren Strukturkonstituenten des verstehensrelevanten Wissens, wenn man so will, die ,kognitiven Frames'. Es wäre dann ein zweiter Schritt einer so verstandenen Semantik, aufzuweisen, wie Wissensrahmen der Teile von ihnen in Strukturen sprachlicher Zeichenketten, verbalisiert' werden. (Busse 2012: 536).

Die Semantik stellt den Ausgangspunkt der epistemologisch ausgerichteten Reflexion von Dietrich Busse dar und wird folglich zur zentralen Bezugsdisziplin bei der Auseinandersetzung mit dem Problem der Wort-, Satzund Textbeschreibung (ders. 2012: u. a. 521 f., 765). Dabei ist die Distanz, ja sogar scharfe Kritik Busses gegenüber dem „schönen strahlenden, makellosen, reinen Modell ,Sprache“" deutlich zu sehen. Die Kritik richtet sich grundsätzlich gegen die sog. „reduktionistischen“ oder „instrumentalistischen" (Zeichen-)Theorien, derer Vertreter nicht in der Lage seien, sich vom wittgensteinschen Erbe loslösen zu können, während die menschlichen Erkenntnisakte bei der Zeichenkonstitution völlig vernachlässigt werden (näher dazu vgl. Busse 2012: 789-794). Von erkenntnistheoretischen Fragen kann sich vor allem die Fachsprachenlinguistik nicht ,befreien'. Ziele, die im 
Rahmen dieser Disziplin verfolgt werden, resultieren u.a. aus der Tatsache, dass Sprache und Erkenntnis unzertrennbar sind.

Lingwistyka języków specjalistycznych musi (...) zdać sobie sprawę z tego, że podstawowych kwestii dotyczących języka nie można rozważać $\mathrm{w}$ oderwaniu od zagadnień dotyczących poznania. (S. Grucza 2008: 96, vgl. auch 2012: 82, 88).

Dass die Erkenntnis durch Sprache und die Sprache durch Erkenntnis geformt werden, steht fest. Deshalb ist der Gegenstand der epistemologischen Semantik, darunter auch der Gegenstand der epistemologischen Fachsprachensemantik so zu profilieren, dass die menschliche Erkenntnisfähigkeit berücksichtigt und die Erkenntnisfaktoren in den Vordergrund gerückt werden, wie dies bereits im Rahmen der Fachsprachenforschung zur Sprache gebracht wurde (vgl. S. Grucza 2008: 195).

Die Erkenntnisfunktionen von Fachsprachen kommen besonders bei der Konstitution von Fachneologismen zum Tragen (vgl. Pawłowski 2015b). Fachwörter, darunter Fachneologismen und genormte Termini sind nicht nur einzig und allein das Ergebnis der menschlichen Erkenntnisakte, sie sind in erster Linie erkenntnisfördernd und helfen das bereits internalisierte Wissen zu organisieren, zu typologisieren, etc. (zu den Funktionen von Termini vgl. F. Grucza 1991: 17-18). Fachneologismen sind außerdem ein motiviertes Beispiel dafür, um zu zeigen, dass sich die Semantiker nicht lediglich auf Bedeutung, wie unterschiedlich sie auch immer konzeptualisiert werden mag, sei es als Designat, Denotation oder Referenz, sei es als Begriff, Sinn oder Konnotation (vgl. Pawłowski 2012: 249), beschränken dürfen. Der Mangel besteht grundsätzlich darin, dass - wie bisher zu lesen ist - Designate, Denotationen, Referenzen etc. nur als Endprodukte, bloß als Ergebnisse der menschlichen Erkenntnis behandelt und analysiert werden, während die Erkenntnis als Tätigkeit, d.h. das Erkennen schlechthin an den Rand der linguistischen Reflexion geschoben wird (vgl. S. Grucza 2012: 77). An Fachneologismen lässt sich dokumentieren, wie irreführend diese Einschränkung ist. Ich schließe mich somit der Auffassung von Sambor Grucza an, der behautet, dass die (anthropozentrische) Fachsprachenlinguistik nicht nur "generative, d.h. textgenerierende Funktionen der Fachsprachen" untersuchen, sondern ihre "kognitiven-wissensgenerierenden Funktionen“ erkennen soll (vgl. ders. 2008: 155). Dass die anthropozentrische Fachsprachenlinguistik an der Erkenntnis, darunter an Fähigkeiten, die sie voraussetzen, interessiert ist, zeigt sich in der Aussage, wie die Fachsprachenkompetenz begriffen wird.

Niezależnie od tego, jak szeroko ujmiemy zakres specjalistycznej kompetencji językowej w każdym przypadku w jej obrębie trzeba wyróżnić umiejętności 
formacyjne (generatywne) oraz funkcyjne. Oznacza to, że specjalistyczna kompetencja formacyjna $\mathrm{w}$ najwęższym rozumieniu języków specjalistycznych to umiejętność tworzenia wyrazów specjalistycznych (terminów), a specjalistyczna kompetencja funkcyjna to zdolność posługiwania się nimi w funkcji znakowej. (S. Grucza 2008: 157).

Die Fähigkeit Fachausdrücke (potenzielle Termini) zu bilden (hier: formative Fachsprachenkompetenz) und diese als Zeichenfunktionen in kommunikativen Akten (hier: funktionale Fachsprachenkompetenz) angemessen einsetzen zu können, liegt allerdings im Ausdehnungsbereich der Fachsprachenlinguistik, gehört aber zugleich m. E. zum enger gefassten Gegenstand der epistemologischen Fachsprachensemantik. Dieser darf keineswegs nur auf Fachneologismen an sich, u. z. ausschließlich auf das Erkenntnis-Ergebnis, reduziert werden, obwohl die Eigenschaften und/oder Funktionen von Fachneologismen durchaus vom Belang sind. Den Gegenstand der epistemologischen Semantik stellen hauptsächlich die epistemischen Eigenschaften und Erkenntnisakte eines Individuums sowie das Bedürfnis dar, diese Erkenntnisakte zu konstruieren ${ }^{3}$ und zu verstehen, wie sie jeweils motiviert sind.

Mit Rücksicht darauf will ich den eigentlichen Gegenstand erläutern und die daraus resultierenden Aufgaben nennen, die die epistemologische Semantik zu examinieren hat. Dabei ist zwischen zwei Forschungsperspektiven, der idiozentrischen und polyzentrischen, zu unterscheiden (s. auch Pawłowski 2012: 260).

\section{idiozentrische Forschungsperspektive}

(1) Mensch in Hinblick auf seine epistemischen und kognitiven Eigenschaften, einzelnen Ausdrücken, ganzen Texten etc. idiolektale Bedeutungen zu zuschreiben,

(2) epistemische Wissensformanten-Typen,

(3) idiolektale (mentale) Ausdrucksform und ihre epistemischen und kognitiven Funktionen; zu trennen ist dabei zwischen der mentalen Ausdrucksform und dem Ausdruck, d.h. einer äußeren Hervorbringung,

3 „Mit Absicht verzichte ich hierfür auf den bislang in der Linguistik weit verbreiteten Terminus "rekonstruieren“, der - so, wie er gebraucht wird - auf die wissenschaftliche Aktivität bezogen wird, die einen Analytiker dazu veranlassen und ihm sogar möglich machen solle, kognitive Inhalte Anderer, unverständlicherweise lediglich aufgrund von Texten, im wahrsten Sinne zu lesen, mit der Überzeugung, eine adäquate aber vor allem wirkliche Rekonstruktion vollziehen zu können, während wirkliche Rekonstruktion erst dann anzunehmen ist, wenn das Muster, nach dem etwas rekonstruiert wird, bereits bekannt oder wenigstens in irgendeiner Form vorgegeben ist." (s. Anm. 6 in Pawłowski 2014: 272). 
(4) idiolektale Bedeutung und ihre epistemischen und kognitiven Funktionen,

(5) epistemisch fundierte Motivation des Sprachelementen; gemeint sind Bedingungen, unter denen idiolektale Bedeutung und idiolektale (mentale) Ausdrucksform miteinander korreliert werden,

(6) innere (mentale) Kontexte, darunter die Kontextualisierung von sprachlichen Elementen im Phrasen-, Satz-, Text- und im allgemeinen epistemischen Wissensrahmen, ${ }^{4}$

(7) polylektale(n) Funktion(en) der Sprachelemente; gemeint sind kognitive Funktionen, die den Sprachelementen im Phrasen-, Satz-, Text- und Wissensrahmen noch auf der Etappe der Äußerungskonstitution ,zugeschrieben' werden,

(8) kulturelle und/oder sprachliche Hervorbringungen, die aufgrund von epistemischen und/oder kognitiven Eigenschaften eines Individuums realisiert werden.

\section{polyzentrische Forschungsperspektive}

(9) kommunikative Funktionen, die den einzelnen bzw. komplexeren Textelementen, in kommunikativen/diskursiven Akten zuteil werden,

(10) äußere (subjektive) Kontexte,

(11) diskursive Rollen der (schriftlichen bzw. mündlichen) Interaktanten,

(12) polylektale Bedeutung einzelner Ausdrücke oder Texte,

(13) polylektale (äußere) Ausdrucksform(en) einzelner Ausdrücke oder Texte.

Auf den ersten Blick wird deutlich, dass der Forschungsgegenstand vor allem im mentalen Bereich positioniert ist. Die mentale Sphäre stellt den eigentlichen Ausgangs- und Bezugspunkt für die epistemologische Semantik dar; den Ausgangspunkt, da der Forscher bei der Datenanalyse den wirklichen Menschen und seine individuelle Erkenntnisperspektive im Auge hat; den Bezugspunkt, da kulturelles und sprachliches Handeln immer zweck- und vor allem subjektorientiert ist. Jede kulturelle/sprachliche Handlung resultiert aus einer Erkenntnisperspektive - sie wird nur aus einer Betrachtungsperspektive ausgeführt, jedoch aus einer anderen identifiziert und verarbeitet. Dabei kann man zwar zwischen Rollen- jedoch nicht zwischen den Erkenntnis- und Betrachtungsperspektiven wechseln. Mittel, die den Interaktanten bei der Kommunikation und den Forschern beim Erreichen der Forschungsziele zur Verfügung stehen, sind - soweit meist zuverlässige -

4 Zur Wissenskontextualisierung vgl. Busse u.a. 2007: 82, 88, zum Wissensrahmen vgl. ders. 2007: 94, 2008a, 70 f., 2012, vgl. auch Ziem 2005: 275 ff. 
empirische Belege in Form von mündlich (Audio- und Videoaufzeichnungen) oder schriftlich abgefassten Texten.

Damit hängen allerdings einige Probleme zusammen. Obwohl Menschen anthropologisch gleich veranlagt sind, so besteht gerade bei der polyzentrischen Forschungsperspektive die Schwierigkeit, analoge Wissenselemente der Idiokultur und die Axiologie mehrerer Individuen empirisch zu identifizieren und nutzbar zu machen. Diese Schwierigkeit gilt als ontologische Hürde insbesondere bei der Deutung der polylektalen Bedeutung (dazu vgl. Pawłowski 2012: 253, Anm. 4). Diese ist das anzunehmende Ergebnis der polylektalen Funktion der in Erwägung gezogenen idiolektalen Bedeutungen. Sie ist dabei ein ,gemeinsames' intellektuelles, ggf. wissenschaftliches Konstrukt, das man ontologisch lediglich im Akzidentellen positionieren kann. Dies gilt auch für die normativen polylektalen Bedeutungen, d.h. Bedeutungen, die bereits sanktioniert sind. Sie sind zum Einen akzidentelle Phänomene, fundiert als wissenschaftliche (hier: lexikographische) Konstrukte, sie ,zeichnen sich' zum Anderen durch eingeschränkte Repräsentativität , aus'. Denn normative Bedeutungen sind nur dermaßen repräsentativ, solange polylektale Funktionen der mit einem Ausdruck korrelierten idiolektalen Bedeutungen analog sind. Die Fragen, was/welche Bedeutungselemente dabei ,die gleichen' sind oder worauf die Kommunikation gestützt ist, sind für die Linguistik und vor allem für die epistemologische Semantik nicht weniger relevant, als herauszufinden, welche Wissensformantentypen sich bei Konstitution einer Äußerung beteiligen. Kurz: die epistemologische Semantik verstehe ich als eine linguistische Teildisziplin, deren Aufgabe ist, (a) den Erkenntnisakten in Bezug auf die Ausdruck- und Bedeutungskonstitution nachzugehen, (b) verstehensrelevante Wissensformanten zu identifizieren, (c) idiolektale Textäußerungen zu beschreiben und (d) diese für die Zwecke der polyzentrisch orientierten Forschung auszuwerten.

Die Aufgaben der epistemologischen Semantik liegen außer den oben genannten auch darin, die epistemische Motivation bei der Ausdruck- und Bedeutungskonstitution zu verstehen. Jeder Linguist, der im Begriff ist, mit dem epistemologischen Ansatz zur Semantik zu arbeiten, soll bemüht sein, alle kognitiven und vor allem epistemischen Eigenschaften zu reflektieren, die diese Motivation formen. $\mathrm{Zu}$ den Aufgaben der epistemologischen Semantik ist deshalb ohne Weiteres die metasemantische Erkenntnisarbeit anzurechnen. Darunter: 1) Wissensaquisition über die epistemischen Wissensformanten-Typen, 2) Wissensaquisition über idiolektale Ausdrucksform und ihre epistemischen und kognitiven Funktionen, 3) Wissensaquisition über die idiolektale Bedeutung und ihre epistemischen und kognitiven Funktionen, 4) Wissensaquisition über epistemisch fundierte Motivation der Sprachelemente, 5) Wissensaquisition über innere Kontexte, 6) Wissensaquisition 
über die polylektalen Funktionen der Sprachelemente, 7) Wissensaquisition über die kulturellen/sprachlichen Hervorbringungen, 8) Wissensaquisition über die kommunikativen Funktionen der Textelemente, 9) Wissensaquisition über die äußeren Kontexte, 10) Wissensaquisition über die diskursiven Rollen der Interaktanten, 11) Wissensaquisition über die polylektalen Bedeutungen der Ausdrücke etc., 12) Wissensaquisition über die polylektalen Ausdrucksformen der Ausdrücke etc., und ,last but not least', 13) Wissensaquisition über die individuellen kognitiven und epistemischen Eigenschaften, die es ermöglichen, jede Erkenntnisarbeit und jedes Erkenntnisergebnis kritisch zu examinieren.

\section{ZUR (FACH-)BEDEUTUNGS- UND (FACH-)AUSDRUCKSKONSTITUTION ${ }^{5}$}

Erkenntnisakte sind bei der Ausdrucks- und Bedeutungskonstitution schlüssig. Dies gilt, wie bereits angedeutet, vor allem für Fachneologismen. Was setzt Erkenntnisakte voraus? Was liegt den Erkenntnisakten zugrunde? Mit anderen Worten: worauf bezieht sich bzw. worauf greift ein durchschnittlicher Sprecher, ein Linguist oder Fachmann zurück, wenn er im Begriff ist, einen neuen Ausdruck oder einen Fachneologismus zu generieren. Welche ,Speisekarte' steht ihm zur Verfügung? Die Frage, wie groß und/oder komplex die einzelnen ,Speisen' sind, kann er aufgrund der Speisekarte selbst nicht beantworten, da sie bloß aus konventionellen Graphemketten zusammengesetzt ist. Wie fein das ,Menü' final ist, hängt in der Tat davon ab, wie raffiniert er bei der Bestellung vorgehen wird. Reichen ihm die Sprachkenntnisse aus, um seinen Geschmackssinn zu befriedigen? Greift er bei der Bestellung lediglich auf die ,usuelle Semantik' bzw. auf die ,usuelle Beschreibung' der Speisen zurück oder sucht er eher ihre wirklichen Designate mental zu aktivieren, die er aus der Erfahrung kennt? Was legitimiert schließlich seine Essbefriedigung: die Sprachkenntnisse oder die epistemische Erfahrung mit dem Referenten? Diese Metapher mag illustrieren, dass Verstehen der Kommunikationsakte und ihre Konstitution nicht durch den Sprachusus allein determiniert sind, sondern vielmehr dank der Erkenntnis zum Tragen kommen. Die Erkenntnis basiert auf der epistemischen Erfahrung, die durch die unmittelbare und mittelbare Wahrnehmung gewonnen wird. Um dies mit Lawrence Barsalou zu sagen, ist die epistemische Erfahrung als „Hinzufügung neuer Exemplare zu den vorhandenen

${ }^{5}$ Zum diesem Problem habe ich mich detaillierter an anderen Stellen geäußert (vgl. Pawłowski 2012, 2013b, 2014). 
Frames [Wissensrahmen]“ $\mathrm{zu}$ deuten, und genauer die „Hinzufügung von neuen Exemplar-Frames zu einem übergeordneten Kategorien-Frame.“ (Barsalou 1992: 64 zit. nach Busse 2012: 392). Die anthropozentrisch ausgerichtete Übertragung dieser Aussage müsste heißen: die Akquisition der Wissenselemente in den/in die bereits internalisierten epistemischen Wissensrahmen.

Man wird sich dabei fragen, ob und wenn ja dann, wie man die epistemische Erfahrung für die Zwecke der semantischen Beschreibung operationalisieren kann. Um dies zu tun, ist es nötig, elementare Erkenntnisparadigmen und methodische Kriterien $\mathrm{zu}$ nennen, die mentale Bereiche ,repräsentieren' und deskribieren lassen. Kriterien, die es möglich machen, nicht nur die Erfahrung schlechthin zu erfassen, sondern auch der Frage nachzugehen, durch welche Faktoren die Bedeutung- und Ausdruckskonstitution geformt wird. Hierfür seien drei epistemische Wissensformantentypen angeführt: (1) der anthropologische, (2) der kulturelle und (3) der axiologische Wissensformantentyp (mehr dazu s. Pawłowski 2012: 256, 2013a: 299-315). Sämtliche Wissenselemente, die sich im Gefolge dieser Formantentypen in der Kognition niederschlagen, sind das Ergebnis der menschlichen Erkenntnisarbeit. Dass ihnen (nach Fähigkeit und Bedarf) epistemische Funktionen ,erteilt' werden können, kann allein die Geschichte der bisherigen Erkenntnisleistungen bezeugen. Die epistemische Funktion der Wissenselemente verursacht es, dass Wissenselemente zu Wissensformanten werden. Das heißt, die Konstitution eines neuen Sprachelements, z. B. eines Fachneologismus, erfolgt erst dann, wenn bereits internalisierte, kognitive Wissenselementen den Status der Erkenntnis-Funktion erreichen (s. auch Pawłowski 2012: 257-259, 2015a: 78).

Wie die empirische Untersuchung zur idiolektalen Bedeutung gezeigt hat (vgl. Pawłowski 2014), ist die Wissenskonstitution epistemisch veranlagt. Reflektiert man idiolektale (fachsprachliche) Bedeutungskonstitution ernsthaft, d.h. wirklichkeitsadäquat und nicht vorprogrammierten Paradigmen zufolge, so lässt sich nicht übersehen, dass sowohl die (fachsprachliche) Bedeutungs-, als auch die Ausdruckskonstitution immer im epistemischen (Fach-)Wissensrahmen erfolgen (vgl. Busse 2007a: 99, 94, 2012, 74, 101), der durch die o.g. Wissensformantentypen, allerdings in unterschiedlichen Proportionen, geprägt ist. Die epistemische (Fach-)Wissensaquisition, und damit die Bedeutungs-, und Ausdruckskonstitution vollziehen sich im inneren Satz-, Phrasen-, Textkontext, werden aber durch den äußeren sozialen, familiären, beruflichen, ethnischen, etc. Kontext (Schroll-Machl/Wiskoski 1999: 55 nach Bonacchi 2011a: 70) und durch diskursive Rollen determiniert (vgl. u.a. Mills, 2007: 16, Radeiski 2011: 157 ff.). Kurz: die (fachsprachliche) Bedeutungs-, und Ausdruckskonstitution erfolgt auf dem Wege der wesent- 
lichen und ggf. weniger wesentlichen Erfahrung mit der unmittelbaren (Fach- bzw.) Alltagswelt, die aus der individuellen Erkenntnisperspektive wahrgenommen und verarbeitet wird - anthropologische, kulturelle und/oder axiologische Wissensformanten sind dabei unumgänglich.

Aus diesem Grund kann und soll die Erfassung der die idiolektale Bedeutungs- und Ausdruckskonstitution konstituierenden (Fach-)Elemente durch apriorische Beschreibungsmodelle prinzipiell nicht eingeschränkt werden. Erkenntnisprozesse sind zwar seit dem Ursprung der Menschheit nach gleichen Prinzipien organisiert, laufen jedoch bei jedem Individuum unterschiedlich ab. Dieses komplexe epistemische Formanten-Menü zu deuten und analytisch auszulegen, heißt zunächst und vor allem die prinzipiellen Schranken der paradigmatischen Semantik zu verlassen und sich nichtreduktionistischen Forschungskonzeptionen $\mathrm{zu}$ zuwenden, welchen die epistemologische Semantik durch und durch gewaschen ist (vgl. Busse 2012: u. a. 791, 795). Dies stellt allerdings eine der größten wissenschaftlichen Herausforderungen dar. Jede idiolektale (Fach-)Bedeutung und jede idiolektale (Fach-)Ausdrucksform werden zum Einen im epistemischen (Fach-)Wissensrahmen als Zeichenelement (Fach-Lexem) konstruiert, welchem unterschiedliche Wissensformantentypen zugrunde liegen, sowie durch einen konkreten (Fach-)Ausdruck (nach außen) vertreten, der für einen beliebigen subjektiv agierenden Interaktanten (vgl. Pawłowski 2013a) und einen weniger subjektiv handelnden Forscher wiederum einen neuen Wissensrahmen eröffnet. Zum Anderen sind ,gemeinsame', oder besser gesagt, analoge bzw. polylektale Bedeutungselemente eines (Fach-)Lexems zu ,erheben', generiert durch die in Erwägung gezogenen Interaktanten, die, um erfolgreich zu kommunizieren, sich auf verstehensrelevante (Fach-)Wissenselemente einigen müssen (vgl. Busse u. a. 2008d, 2012: 23, 34, 76). ${ }^{6}$ Sie sind mit anderen Worten genötigt, ihre individuellen (Fach-)Bedeutungen auszuhandeln. Und das determiniert wiederum einen neuen epistemischen (Fach-)Wissensrahmen.

\section{ZUR METHODIK}

Die Erkenntnisziele und damit die empirische Analyse der epistemologischen Semantik sind mit Blick auf die menschliche Erkenntnisarbeit ausgerichtet. Es handelt sich hier konkret um eine Studie zur Konstitution der

${ }^{6}$ „Der kognitiven Semantik geht es dagegen mehr um die Strukturen, die das verstehensermöglichende Wissen annimmt, und um die kognitiv-epistemischen Prozesse, die für die jeweils neu zu vollziehende Konstitution bzw. Realisierung von sprachzeichengestütztem Sinn eine notwendige Voraussetzung sind." (Busse 2008c: 16). 
Fachneologismen, mit der ich Mitte Mai 2014 begonnen habe. Empirisches Material der Studie sind polnische Translate der Fachtexte im Fachgebiet ,Altgriechische und Byzantinische Musikologie' (im Einzelnen: Martin L. West, 2003. Muzyka starożytnej Grecji. Kraków; John G. Landels, 2003. Muzyka starożytnej Grecji i Rzymu, Kraków; Egon Wellesz, 2006. Historia muzyki i hymnografii bizantyjskiej, Kraków). Die Methodik ist idiozentrisch profiliert. Hier liegt also das induktive Verfahren vor, mit dem aus Prinzip keine repräsentative Erhebung erstrebt wird. Die methodischen Grundsteine der Studie sind so zusammengestellt, um die Daten über die Erkenntnisakte zu gewinnen. Das sind: Intensivinterview als Leitfadengespräch mit dem Innovator/Übersetzer (Maciej Kaziński). Empirische Ziele sind die Translatanalyse und die Evaluationen der Interviews. Bisher wurden acht Interviews durchgeführt, wobei das letzte am 16. Februar 2015. (Zur Methodik der Interviews s. Pawłowski 2015c).

\section{ZUR ANALYSE DES FACHNEOLOGISMUS ,AULETA`}

Eine exemplarische Analyse des zu Beginn angesprochenen Fachneologismus auleta will ich aufgrund der o.g. Fachtexte durchführen. Ergebnisse über den Erkenntnisakten des Innovators/Übersetzers liegen vor (s. Pawłowski 2015b). Sie wurden anhand eines zu diesem Zweck initiierten und aufgezeichneten Interviews vom 20. Mai 2014 gewonnen. Auleta entspricht bei Landels (1999) und West (1992) bis auf einige Ausnahmen7 seinem englischen Äquivalent, Aulete' [IPA: s:li:t], das für den Innovator/Übersetzer ein Ausgangsterminus darstellt. ,Aulete' geht auf das griechische $\alpha \dot{\lambda} \lambda \dot{\eta} \tau \eta \varsigma$ zu-

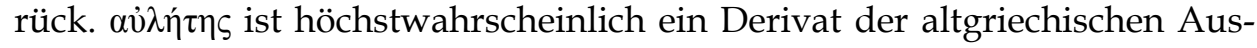

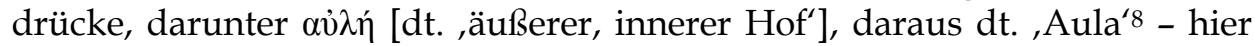

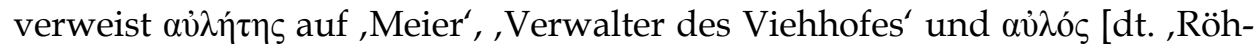

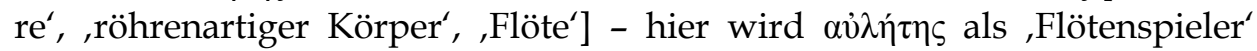
übersetzt (vgl. Frisk 1960: 186-187). Aus der lexikographischen Analyse geht

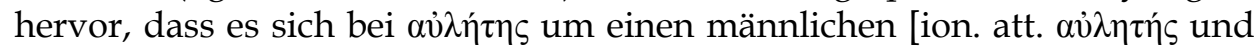

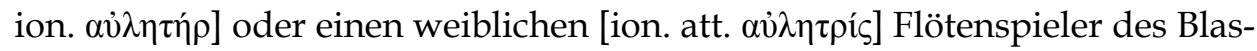

7 West (1961) wechselt allerdings im englischen Originaltext zwischen ,aulete' und ,piper', im Gegensatz zu den polnischen Übersetzern, die sich konsequent des Zielterminus ,auleta' bedienen. Landels (1999) dagegen gebraucht hierfür den analytischen Fachausdruck ,aulosplayer', während , auletes' nur einmal als Transkription aus dem Griechischen vorkommt.

8 „Aula, F., »Veranstaltungsraum«, (M. 16. Jh. »künstliche Macht, Hofstaat«,) 17. Jh. (1617) Lw. lat. aula, F., »Atrium, Halle«, Lw. gr. aulé, F., »Hof, Platz vor der Wohnung«" (Köbler, 1995: 31). 
instrumentes a $\lambda \lambda$ ó $\varsigma$ handelt. ${ }^{9}$ Der Aulos ist ein röhrenartiger Körper, eine

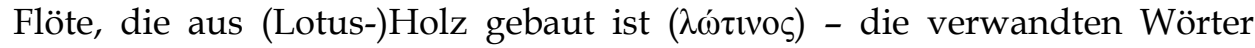
verweisen ebenfalls auf Holz, als Baustoff des Instruments: lit. aũlas ,Stiefelschaft', nnorw. aul ,der hohle Stengel der Angelica', lat. alvus ,Höhlung', lit. aulỹs, aksl. ulojb ,Bienenstock' (eig. ,hohler Baumstamm'); apr. aulis ,Schienbein', aulinis ,Stiefelschaft' (vgl. Frisk 1960: 186-187). Der Aulos wird geblasen ( $\alpha \dot{\lambda} \lambda \dot{\varepsilon} \omega)$ oder einfach gespielt $(\lambda \omega \tau \dot{\varepsilon} \omega)$ (vgl. Frisk 1970: 153). Es ist anzu-

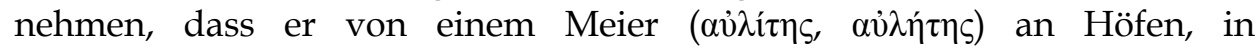

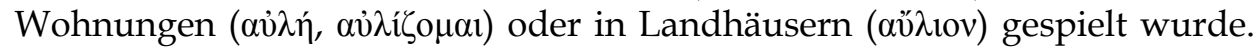
Der Klang dieses Instruments müsste dabei lärmig ( $(\tau \dot{\rho} \rho \beta \eta)$ sein (vgl. Frisk 1970: 947) und für (geistige) Verwirrung sorgen (vgl. West 1961: 105-106).10

Das vorläufige Ergebnis der Analyse lässt schlussfolgern, dass es sich bei der Konstitution des Derivats $\alpha \hat{\lambda} \lambda \eta \dot{\tau} \tau \varsigma$ um eine Reihe von unterschiedlichen Bedeutungs-Formanten gehandelt haben musste, die nicht nur Eigenschaften des Referenten, darunter sein Aussehen (visuelle), Baustoff (takti-

\footnotetext{
9 The New Grove Dictionary of Music and Musicians gibt dazu folgende Erläuterungen an: "An 'aulete' (Gr. aulètēs; Lat. tibicen) was a male player, either amateur or professional, of the aulos; 'auletride' (aulētris) was the name for a female player of the instrument. (...) The single, generic term aulètēs was used for the highly esteemed virtuoso players who gave recitals and also competed against each other, especially at sacred festivals. However, at the beginning of the Hellenistic period (the last quarter of the $4^{\text {th }}$ century BCE), other, more specific terms began to appear in connection with two musical genres - the solo aulos and the aulos with chorus, both of which were originally performed by auletes until they decided to specialize in one or the other category." (Sadie/Tyrrell 2001: 182).

10 „Aulos był znany ze zdolności wyrażania i pobudzania różnych emocji. Dawne utwory przypisywane Olymposowi, nawet w złym wykonaniu, miały według Platona moc doprowadzania słuchacza do szału, tak że musiał poddać się religijnemu oczyszczeniu. Arystoteles nazywa aulos ,orgiastycznym', czyli prowadzącym do szału religijnego; zazwyczaj wymienia się go (wraz z bębenkami) w związku z kultem bakchicznym, korybanckim i podobnymi kultami ekstatycznymi. Słyszymy o mężczyźnie, którego wpędzał w panikę dźwięk aulosów podczas uczty. ,Longin' mówi, że aulos pozbawia słuchaczy rozumu i każe ich stopom wybijać rytm. Może jednak również - gdy zagrać na nim poważnie i słodko - wypełnić duszę spokojem. Doświadczony auleta spełni każde życzenie: może uśmierzyć ból, rozbudzić radość, rozpalić kochanka, uwznioślić pobożnego.” (West 2003, 121). „The aulos was noted for its ability to express and to arouse different emotions. The old aulos-tunes attributed to Olympus, even if badly played, according to Plato, have the power to possess the hearer with frenzy and mark him out as being in need of religious purification. Aristotle calls the aulos 'orgiastic', i.e. conducive to religious frenzy, and it is regularly mentioned (together with drums) in connection with Bacchic, Corybantic, and suchlike ecstatic cults. We hear of a man whom the sound of auloi at the symposium always affected with panic.104 'Longinus' speaks of the aulos sending the listeners out of their minds and setting their feet tapping to the rhythm. But it can also fill the soul with calm if it is played soberly and sweetly. The expert aulete can provide whatever is wanted: he can assuage grief, enhance joy, inflame the lover, exalt the devout." (West 1961: 105-106).
} 
le/haptische), Klang (auditive, proxemische, emotive, axiologische), sondern auch die Spielart (motorisch-kinetische, sogar gustatorische) und nicht zuletzt seine kulturellen und kommunikativen Funktionen (propriorezeptive) erkennen lassen. Diese Bedeutungsformanten gehen jedoch mitsamt der Konstitution des polnischen Fachneologismus auleta zum Teil verloren. ${ }^{11}$ Die Gründe sind verschieden. Zum einen wird durch die Transliteration die Graphemik geändert und dadurch die Morphemik beeinträchtigt. Zum anderen tritt auleta bis auf seine unmittelbaren morphologischen,Verwandten' aulos im Fachzieltext völlig isoliert auf, ohne dass semantisch-syntaktische Relationen des Derivats $\alpha \dot{\lambda} \lambda \eta \dot{\tau} \eta \varsigma$ und vor allem Bezüge zu den Wortwurzeln

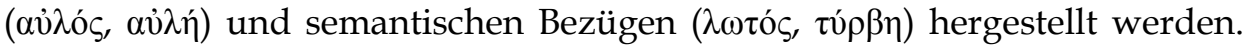
Mit dem Zielterminus schwindet sozusagen das ursprüngliche ,Menü’ der epistemischen Erfahrung definitiv dahin.

Auf welche epistemische Erfahrung/auf welche kognitiven Ressourcen, greift der Innovator/Übersetzer zurück, der in der Not ist, einen polnischen Fachneologismus einführen zu müssen. auleta kommt an mehreren Stellen im Fachtext vor (vgl. Landels 2003: u.a. 41-63, West 2003: u.a. 97-122).12 Der

${ }^{11}$ Landels (1999) rechtfertigt explizite die Entscheidung, warum er es für nötig hält, den Termins aulos oder das lateinische tibia statt flute [pln. flet] oder einen recht umständlichen analytischen Fachausdruck reed-blown double pipe [pln. podwójna piszczatka podwójnostroikowa] zu gebrauchen. "So it is easy to show that the words aulos and tibia should not be translated as ,flute'; but to suggest a good alternative is not so easy, because there is no well-known instrument in modern use which corresponds on all three points-a double pipe, with double reeds and a cylindrical bore. In Europe the technique of double-piping survives only in folk music of remote regions, and to translate aulos by the name of (say) some Caucasian double pipe would be to explain the unfamiliar in terms of the unheard-of. For a technically accurate prosaic translation 'reed-blown double pipe' is perhaps the best available; for less exact requirements 'pipes' will do, given an explanatory footnote for Scottish readers that bagpipes are not indicated. In this book from now on, the instrument will be called aulos, which is to be regarded as a loan-word; but I shall retain the Greek plural auloi, as being less troublesome than ,aulosses'." (ders, 25). „Łatwo zatem wykazać, że słowa aulos i tiba nie powinny być tłumaczone jako ,flet', trudno jednak zaproponować dobrą alternatywę, gdyż nie istnieje żaden znany i używany dzisiaj instrument, który odpowiadałby wszystkim trzem cechom aulosu - posiadał mianowicie podwójną piszczałkę, podwójny stroik i cylindryczny kanał. W Europie technika gry na dwóch piszczałkach występuje tylko w odległych kulturach ludowych, więc opisywanie aulosu poprzez nazwę jakiegoś - powiedzmy - kaukaskiego instrumentu byłoby tłumaczeniem ignotum per ignotum. Opisując rzecz w kategoriach technicznych, użylibyśmy precyzyjnego określenia ,podwójna piszczałka podwójnostroikowa', jednak w książce tej będziemy odtąd używać słowa aulos jako nazwy własnej." (Landels 2003: 42).

12 Deklinierte Neubildungen auleci, auletów, auletę sind bereits durch die polnische Übersetzung von The Rise of Music in the Ancient World von Curt Sachs bekannt. Die Übersetzerin, Zofia Chychlińska, gebraucht die o.g. Formen, ohne sich explizite auf die Nominativform auleta zu beziehen. Man kann jedoch annehmen, dass sie diese wenigstens gedacht hat (vgl. 
Innovator/Übersetzer folgt bei der Konstitution dieses Fachneologismus den analogen, jedoch nicht sehr üblichen, polnischen Ausdrucksformen wie poeta, autleta, geodeta, die jedoch nicht dem Musikbereich entstammen. Eliminanten, die Maciej Kaziński im Interview genannt hast, sind aulecista, aulista und auletnik. Übliche und vor allem meist wahrscheinliche Alternativbildung, die Musiker bezeichnen flecista, lutnista perkusista, organista, gitarzysta, chórzysta aber auch Berufsbezeichnungen archiwista, ekonomista, iluzjonista, kombajnista, maszynista, urbanista stellen meiner Meinung nach ein durchaus passendes morphologisches Paradigma dar. Kaziński folgt jedoch

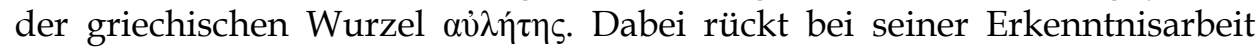
nicht nur der Wortstamm av̉ $\lambda$ - schlechthin 13 , sondern auch die Ableitung -

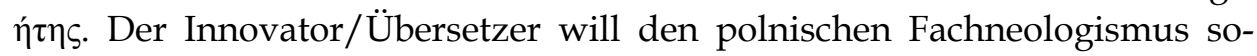
weit wie möglich an die griechische Kulturquelle binden, während er das morphologische Wortbildungsmuster -ista als zu modern und als Zeit ungemäß verwirft. (Mehr zu den Ergebnissen der Analyse des Interviews siehe Pawłowski 2015b). Dem gleichen Wortbildungsmuster folgt die Koübersetzerin, Anna Maciejewska. aulodowie und kitarodowie verweisen auf $\grave{\emptyset} \delta \dot{n}$

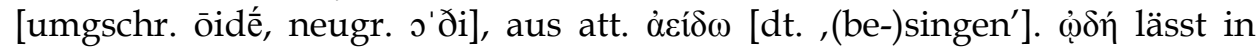
auloda, kitaroda einen Gesang, und gezielt, einen Sänger erkennen, der zu

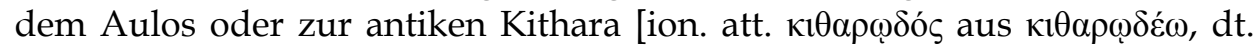
,Zithersänger', ,zithersingen'] gesungen hat (West 2003: 33, vgl. auch Frisk 1960: 850-851).

Außer der morphologischen Arbeit greift der Innovator zur griechischen Ikonografie und aktiviert visuelle Wissensformenten. Er lässt außerdem auditive, emotive, motorische oder axiologische Formanten ,mitspielen', indem er sich Rekonstruktionen der antiken Musik anhört ${ }^{14}$ und damit analogen Bedeutungselemente des griechischen Derivats $\alpha \dot{\nu} \lambda \hat{\eta} \tau \eta \varsigma$ wenigstens teilweise wiederherstellt.

\section{ZUR FACHTEXTANALYSE}

Wie verhält sich auleta im polnischen Fachtext? Um die Frage zu beantworten, mögen exemplarisch Textausschnitte aus Martin West examiniert

Sachs 1988: 209, 227, 283, 297, 299). Kaziński hat im Interview zugegeben, dass er den polnischen Translat zwar gelesen, jedoch nicht für inspirierend gehalten hat.

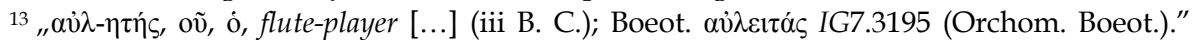
vgl. The Online Liddell-Scott-Jones Greek-English Lexicon

${ }_{14}$ Bekannt dafür ist Stefan Hagel vom Institut für Kulturgeschichte der Antike der Österreichischen Akademie der Wissenschaften <http://homepage.univie.ac.at/stefan.hagel/ und http://www.oeaw.ac.at/kal/agm/>. 
werden. Der erste Ausschnitt lässt einen tanzenden Chorkreis von fünfzig Menschen erkennen, in dessen Mitte sich der Aulet befindet. Każdy chór liczyz pięćdziesiąt osób, tańczących w kolistym układzie, z auletą pośrodku. (West 2003: 31). ${ }^{15}$ Das erste Textexzerpt lässt folgenden Wissensrahmen des Fachworts auleta herstellen (siehe Abb. 1). Zu sehen sind das Agens, chór' [Chor] und die Attribute ,tańczą' [tanzen], ,w kole' [im Kreis], ,pięćdziesiąt' [fünfzig]. Die Beziehung ,chór' und ,auleta' ist "bislang“ einseitig; der Chor tanzt um den Auleten herum, während er keine Handlung vollzieht.

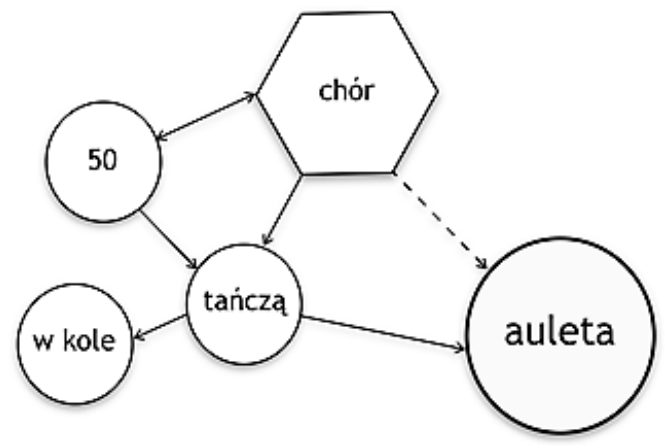

Abb. 1. Wissensrahmen auleta ${ }^{16}$

Goście Agatona na uczcie, która opisat Platon, rezygnuja z ustug auletki , niech sobie samej gra, jeżeli ma na to ochote, albo niewiastom tam, w dalszych pokojach. (West, 2003: 40).17 Daraus resultieren weitere Elemente des Wissensrahmens (siehe Abb. 2.): das Agens ,goście Agatona' [Agathons Gäste], die Prädikation ,niech gra' [soll spielen], die Attribute ,rezygnują' [verzichten], ,uczta' [Gastmahl], ,niewiastom' [den Weibern], ,(inne) niewiasty' [(andere) Weiber], , w' [in], ,dalszych pokojach' [in weiteren Zimmern] und die metatextuelle Präsupposition ,Platon'. Es ist übrigens unklar, in welchem Verhältnis das Attribut ,niewiastom' und die Präsupposition ,(inne) niewiasty' zueinanderstehen. In den Vordergrund rückt vor allem die weibliche Form auletka. Damit ist deutlich gesagt, dass Aulos unmissverständlich beide Geschlechter gespielt haben.

15 "Each chorus was fifty strong, dancing in circular formation with its piper in the middle." (West 1992: 16-17).

${ }^{16}$ Deskriptive Regeln nach Dietrich Busse (2012: 786).

17 "Agathon's guests at the party described by Plato decide to dispense with their piper's services and 'let her go and pipe to herself or, if she likes, to the women inside." (West 1992: 26-27). 


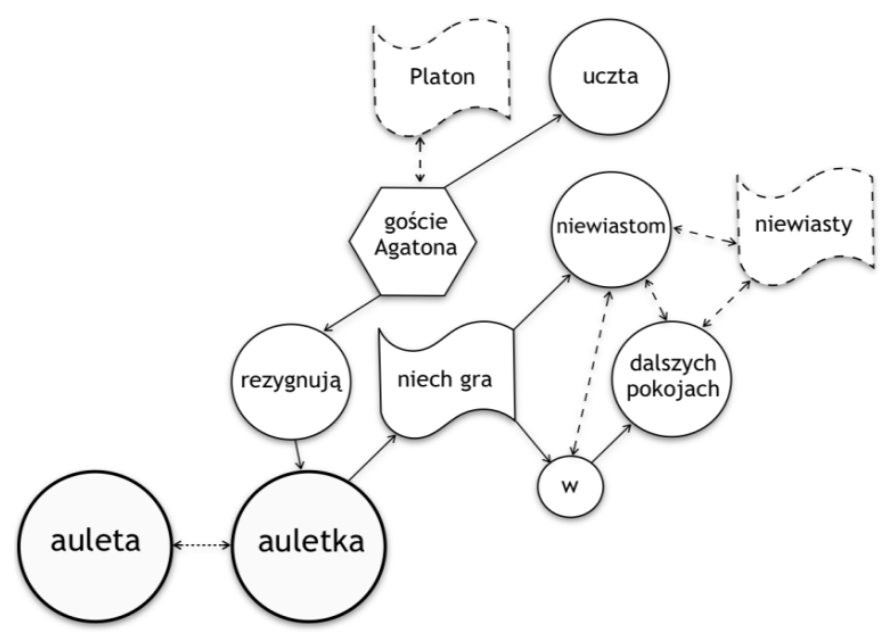

Abb. 2. Wissensrahmen auleta und auletka

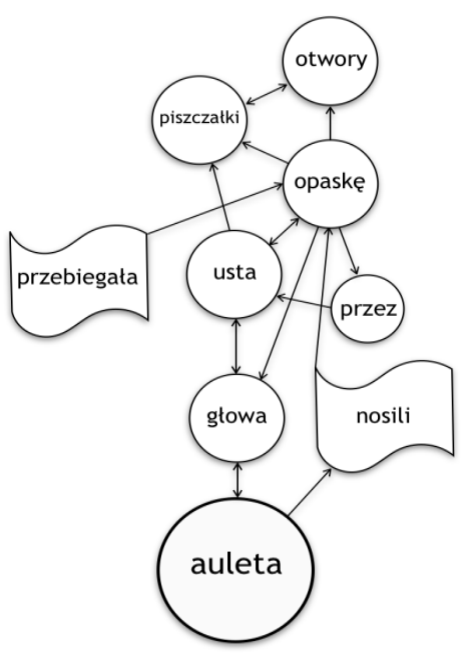

Abb. 3. Wissensrahmen auleta

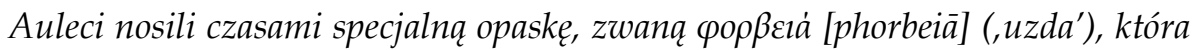
przebiegata przez usta ( $z$ otworem lub dwoma otworami na piszczałki) ku tyłowi glowy (...).18

18 "The aulete sometimes wore a special kind of strap, called a phorbeia ('halter'), that went across his mouth (with a hole, or two holes, for the pipes) and round the back of his head (...)." (West 1961: 89). 
Aus diesem Textausschnitt gehen weitere Elemente hervor, die den Wissensrahmen auleta zunächst um die Prädikationen ,nosili' [trugen] und ,przebiegała' [ging] erweitern lassen (siehe Abb. 3.). Der Ausschnitt beinhaltet außerdem viele Attribute: ,głowa' [Kopf], ,usta' [Lippen], ,opaskę [Band], ,przez' [um], ,otowry' [Löcher], ,piszczałki' [Pfeifen].

Das folgende Exzerpt lässt weitere Elemente des Wissensrahmens an den Tag kommen. „Maja około 20-25 cm długości. Prawdopodobnie takich rozmiarów były w swej petnej postaci dwa archaiczne aulosy z Efezu. Większość instrumentów wyglada na póttora raza dłuższe, niektóre na dwa razy dłuższe. Zachowane aulosy (poza tymi z Efezu), które sa w stanie pozwalajacym na dokonanie pomiarów, maja po $30 \mathrm{~cm}$ dtugości i więcej; najdtuższe (49-57) sa starannie wypracowane instrumenty z Pompejów. Pauzeniasz pisze, że stynny auleta argiwski Sakadas trzymał na posąu parę aulosów o dtugości równej własnemu wzrostowi."19 (West 2003: 106). $\mathrm{Zu}$ erkennen sind das Agens, auleta', die Prädikationen ,trzymał' [hielt], die Attribute ,aulos', ,dwa aulosy' [zwei Auloi], ,zachowane aualosy' [überlieferte Auloi], ,większość instrumentów ${ }^{\star}$ [die meisten Instrumente], ,Pompejów ${ }^{\prime}$

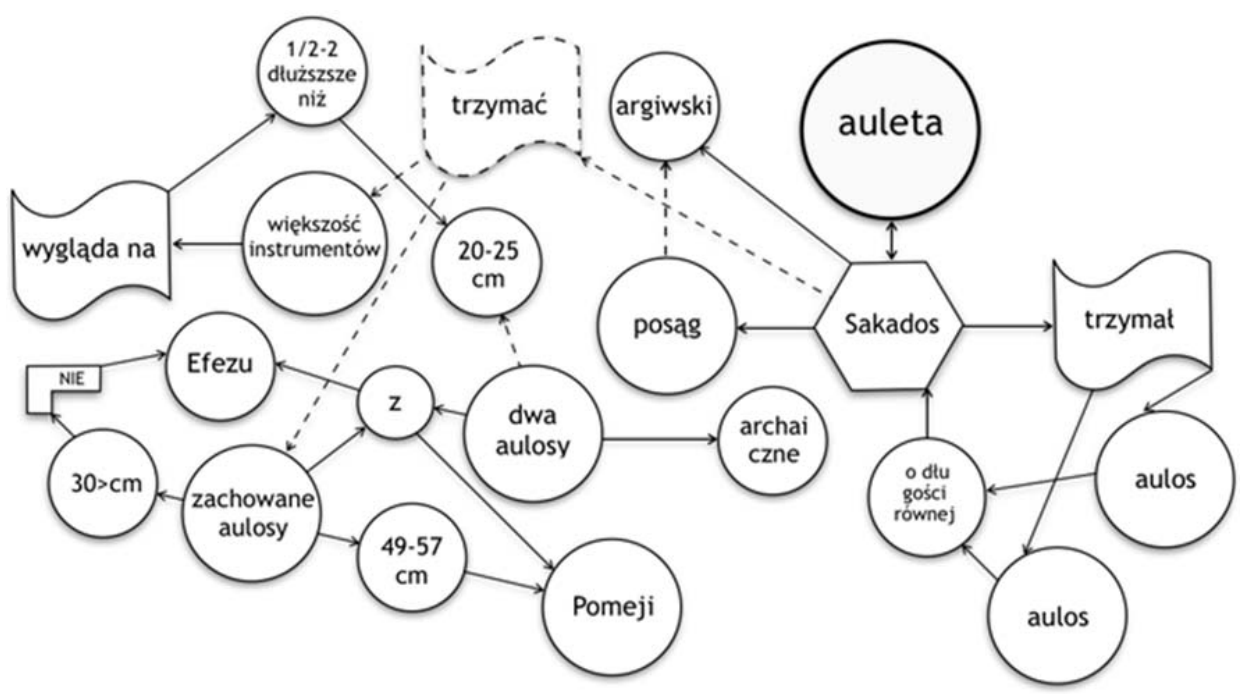

Abb. 4. Wissensrahmen auleta

19 "The length suggested is about 20-25 cm. (8- 10 inches). The two Archaic auloi from Ephesus were probably about this size when complete. Most instruments look at least half as long again, and some twice as long. Apart from the Ephesian ones, the surviving auloi, where complete enough to be measured, are of $30 \mathrm{~cm}$. upward, the longest being the elaborate instruments from Pompeii $(49-57 \mathrm{~cm}$.). Pausanias mentions a statue of the famous Argive aulete Sakadas with a pair of auloi as tall as himself." (West 1961: 90). 
[Pompei], ,posąg' [Statue], ,Sakados', ,o długości równej' [mit der Länge von], , z' [aus], einige Werte ,20-25/30>/49-57 cm', archaiczne' [archaische], ,argiwski‘ [argivisch], ,półtora/dwa razy dłuższe' [anderthalb/zwei Mal länger] sowie eine Präsupposition ,trzymaćc [halten] und ein Negationsoperator ,nie ${ }^{\prime}$ [nein]. Dem Text nach erhalten wir folgenden Wissensrahmen (siehe Abb. 4.).

Mit weiteren Exzerpten wird der Wissensrahmen selbstverständlich immer umfangreicher werden. Die hier zitierten Textausschnitte lassen einen Wissensrahmen erstellen (siehe Abb. 5.), in dem nicht nur die polylektale Bedeutung des Terminus an/für sich erfasst ist (vgl. Pawłowski 2010: 253, 2014, 285), sondern viel mehr , seine' syntaktischen Relationen mit anderen Fachausdrücken und/oder Begleitwörtern wiedergegeben sind (vgl. Busse 2012: 443). Ans Licht kommen nicht nur die Eigenschaften des Gegenstands ,aulos', u. a. Länge, Bestandteile, sondern auch seine (kulturellen und kommunikativen) Funktionen, z. B. die Unterhaltungsfunktion, die in der Fachtextsyntax zu erkennen sind.

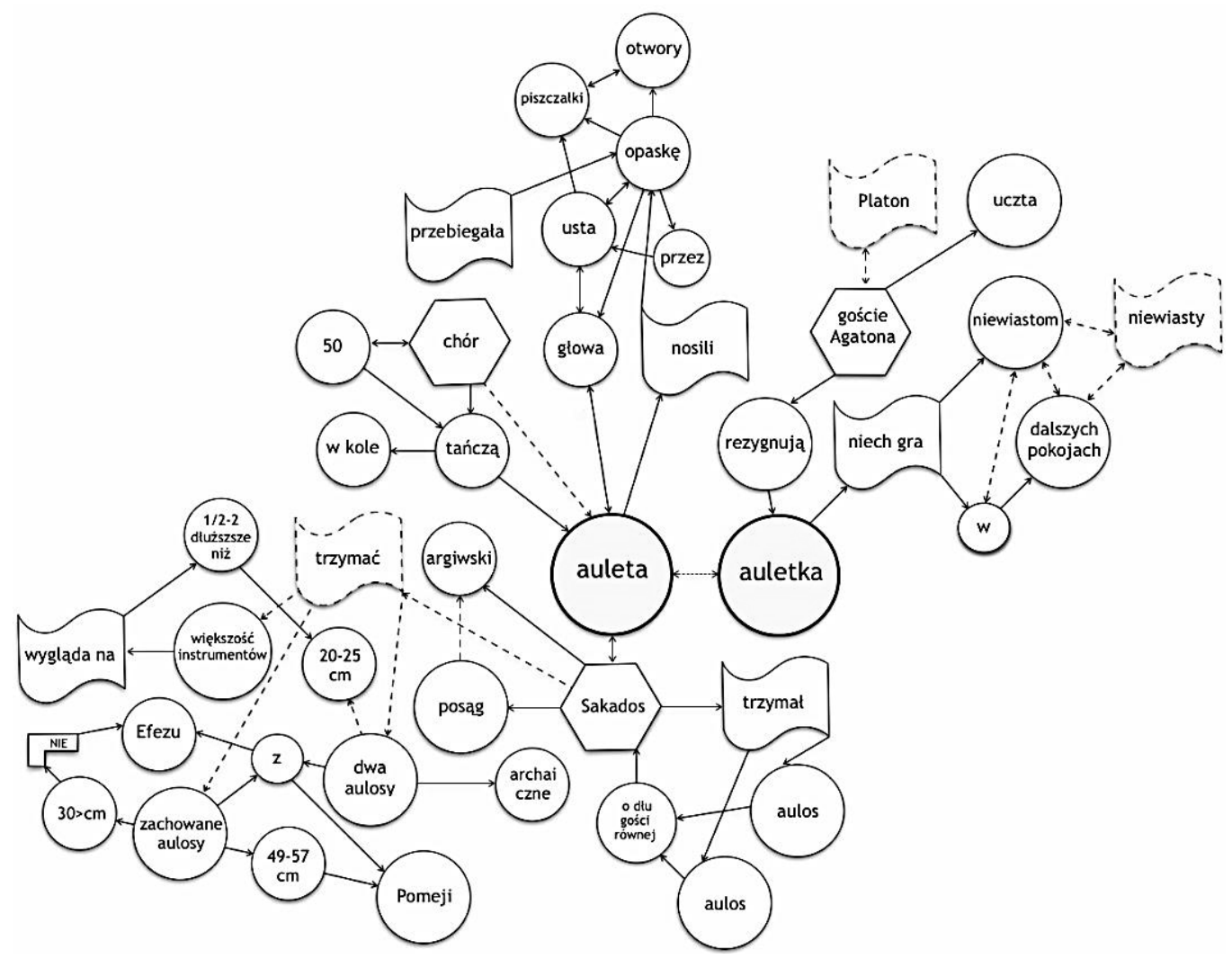

Abb. 5. Gesamt-Wissensrahmen auleta und auletka 
Die zitierten Exzerpte des polnischen Fachtranslats lassen ein durchaus wenig raffiniertes ,Menu' der Bedeutungsformenten erkennen, die sich an der Konstitution des Fachneologismus auleta mögen beteiligt haben; zu nennen sind die visuellen, auditiven und die motorischen. Diejenigen Wissensformanten, die dem griechischen Derivat aủ $\lambda \dot{\eta} \tau \eta$, zu entnehmen' sind, wie etwa die emotiven, propriorezeptiven, axiologischen, etc., werden allein nach der Lektüre des Fachtranslats eher nicht aktiviert. Wie oben angesprochen, ist der Innovator/Übersetzer in seiner Entscheidung eher eingeschränkt. Er ist durch die Morphologie des Ausdrucks auleta eingeschränkt, kann allerdings, ja er soll sogar die Fachdesignate wahrnehmen und verarbeiten. D.h. er soll m. E. alle nötigen Wissensfomantentypen aktivieren, bevor er seine Erkenntnisarbeit am Zielterminus endgültig abschlossen hat.

\section{RÜCKBLICK UND AUSBLICK}

Erste Schritte, die ich in Richtung epistemologische Semantik unternommen habe (vgl. Pawłowski 2007, 2009, 2010, 2011, 2012, 2013a/b, 2015a/b), basierten auf der anthropozentrischen Linguistik und Kulturologie (vgl. Jan Niecisław Baudouin de Courtenay 1904, 1909, F. Grucza u.a. 1983, 1994, 2000, 2010, 2012a/b, S. Grucza u.a. 2008, 2010, 2012, 2013, S. Bonacchi u.a. 2009, 2010, 2011a/b) sowie auf der Frame-Semantik (vgl. D. Busse v.a. 2012). Langsam wurde mir klar, wie stark die Ausdrucks- und Bedeutungskonstitution von der epistemischen Erfahrung abhängt. Meine Bemühungen, dies ausschließlich an Texten, darunter an Audio-Korpora gesungener Texte, empirisch zu dokumentieren (vgl. Pawłowski 2014), wurden leider nur zum Teil von Erfolg gekrönt. Es hat sich herausgestellt, dass Textäußerungen ein unzureichendes empirisches Material darstellen, um die Erkenntnisarbeit eines Textproduzenten, der empirisch nicht mehr zu erreichen ist, genügend nachvollziehen zu können. Außerdem war und ist es soweit schwer, die epistemischen Akte an bereits fixierten, gängigen oder teilweise veralteten Ausrücken empirisch zu belegen und zu erläutern. Priorität war, ein passendes Textmaterial und eine akkurate Methode zu finden, bzw. diese zu entwickeln. Fachneologismen eröffnen hierfür einen großen Spielraum zur Analyse. Dabei bieten das Intensiv- und Leitfadengespräch bei der Auseinandersetzung mit dem Problem ,Erkenntnisakte des Innovators/Übersetzers' ein adäquates und effizientes Instrument.

Die epistemologische Semantik ist eine Disziplin, die zweierlei gewährleistet. Sie schafft zum Einen eine Umorientierung sowohl bei der Deutung der Ausdruck- und Bedeutungskonstitution, als auch bei der Analyse der idiolektalen Textäußerungen und ihrer polylektalen Geltung. Die idiozentri- 
sche und polyzentrische Forschungsperspektive liefert hier eindeutige wissenschaftliche Positionierung. Die epistemologische Semantik schafft zum Anderen eine Umorientierung bei der Erfassung des Forschungsgegenstandes. Er ist gegenüber seinen Idealisierungen wirklichkeitsadäquat. Konkrete Menschen mit konkreten individuellen Eigenschaften und konkreter einmaliger epistemischer Erfahrung erfüllen einwandfrei das Kriterium der adäquaten Annahmen. Mag die Forschungsperspektive variieren, so steht die menschliche Erkenntnisarbeit definitiv im Mittelpunkt des Forschungsinteresses der epistemologischen Semantik. Linguistische Erkenntnisakte und Erkenntnisergebnisse stellen den enger gefassten Forschungsgegenstand dar. Daraus resultieren steigende Anforderungen an die Vertreter der Semantik, die sich ständig mit neuen Konstitutionsmustern und neuen, ständig variierenden epistemischen Wissensrahmen auseinandersetzen müssen. Sie sind dabei nicht hilflos. Klare Bezüge zur analogen Ausdrucks- und/oder Textkonstitution und -interpretation (siehe "verstehensrelevantes Wissen" bei Busse 2012: 805-806) und vor allem die gleiche menschliche Veranlagung (siehe "anthropozentrische Linguistik“), die sich in anthropologischen, kulturellen und axiologischen Wissensformantentypen manifestiert, machen die Aufgabe wesentlich leichter.

Die epistemologische Semantik, in der Art und Weise, wie sie hier betrieben wird, eröffnet eine Palette alternativer Forschungslösungen für diverse linguistische Fachrichtungen, zum Beispiel für die Translationswissenschaft. Translatorische Implikationen, die den Ergebnissen der Studie zu entnehmen sind, liegen auf der Hand (Pawłowski 2015b/c). Der , altgriechische' Fachneologismus auleta, wie ungewöhnlich er auch immer klingen mag, stellt lediglich eins der reichlichen Exempla in diesem Bereich.

\section{LITERATURVERZEICHNIS}

Abraham, W. / Binnich, R. 1972. Generative Semantik. Frankfurt/M.: Athenäum.

Apresjan, J. D. 2000. Semantyka leksykalna. Synonimiczne środki jezyka. Wrocław / Warszawa / Kraków: Ossolineum.

Baudouin de Courtenay, Jan Niecisław F. 1909. Zarys historji językoznawstwa czyli lingwistyki (glottologji). Warszawa: Drukarnia Naukowa, <www.polona.pl>.

Baudouin de Courtenay, Jan Niecisław F. 1904. Szkice Jezzykoznawcze. Bd. 1. Warszawa: P. Laskauer, <www.polona.pl>.

Bärenfänger, O. 2002. Merkmals- und Prototypensemantik: Einige grundsätzliche Überlegungen. In: Linguistik online 12, 3/02 <http://www.linguistik-online.de/12_02/baerenfaen ger.html>, 3-17.

Beeh, V. 1973. Ansätze zu einer Wahrheitswertfunktionalen Semantik. München: Hueber.

Birwisch, M. 1983. Psychologische Aspekte der Semantik natürlicher Sprachen. In: Matsch, W. / Viehweger, D., (Hrsg.). Richtungen der modernen Semantikforschungen. Berlin: AkademieVerlag, 15-64. 
Bock, H. 1990. Semantische Realität. Beitrage zu einer psychologischen Bedeutungslehre des Sprachgebrauchs. Göttingen: Hogrefe.

Bonacchi, S. 2011a. Höflichkeitsausdrücke und anthropozentrische Linguistik. Warszawa: Euroedukacja.

Bonacchi, S. 2011b. Anthropozentrische Kulturologie: Einige Überlegungen zu Grundannahmen und Forschungspraxis anhand der Analyse von Komplimenten. In: Grucza, F. / Pawłowski, G. / Zimniak, P. (Hrsg.). Die deutsche Sprache, Literatur und Kultur in polnischdeutscher Interaktion. Warszawa: Euroedukacja, 33-52.

Bonacchi, S. 2010. Zum Gegenstand der anthropozentrischen Kulturwissenschaft. In: Lingwistyka Stosowana / Applied Linguistics, 2, 69-81.

Bonacchi, S. 2009. Zur Vieldeutigkeit des Ausdrucks Kultur und zur anthropozentrischen Kulturtheorie. In: Kwartalnik Neofilologiczny, 56, 1, 25-45.

Busse, D. 2012. Frame-Semantik. Ein Kompendium. Berlin, Boston: de Gruyter.

Busse, D. 2009. Textbedeutung und Textverstehen aus Sicht einer linguistischen Epistemologie. In: Bachmann-Stein, A. / Merten, S. Roth, Ch. (Hrsg.). Perspektiven auf Wort, Satz und Text. Semantisierungsprozesse auf unterschiedlichen Ebenen des Sprachsystems. (=Festschrift für Inge Pohl) Trier: WVT Wissenschaftlicher Verlag Trier 2009, 45-56. <http://www.phil-fak.uniduesseldorf.de/fileadmin/Redaktion/Institute/Germanistik/AbteilungI/Busse/Texte/Bus se-2009-02-ur.pdf $>$.

Busse, D. 2008a. Diskurslinguistik als Epistemologie. Das verstehensrelevante Wissen als Gegenstand linguistischer Forschung. In: Warnke, I. / Spitzmüller, J. (Hrsg.). Methoden der Diskurslinguistik. Sprachwissenschaftliche Zugänge zur transtextuellen Ebene. Berlin: de Gruyter, 57-88. <http://www.phil-fak.uni-duesseldorf.de/fileadmin/Redaktion/Institute/Germa nistik/AbteilungI/Busse/Texte/Busse-2008-03-ur.pdf>.

Busse, D. 2008b. Begriffsgeschichte - Diskursgeschichte - Linguistische Epistemologie. Bemerkungen zu den theoretischen und methodischen Grundlagen einer Historischen Semantik in philosophischem Interesse anlässlich einer Philosophie der Person. In: Haardt, A. / Plotnikov, N. (Hrsg.). Diskurse der Personalität: Die Begriffsgeschichte der ,Person' aus deutscher und russischer Perspektive. München: Wilhelm Fink, 115-142. <http://www.philfak.uni-duesseldorf.de/fileadmin/Redaktion/Institute/Germanistik/AbteilungI/Busse/ Texte/Busse-2008-04-ur.pdf >.

Busse, D. 2008c. Linguistische Epistemologie. Zur Konvergenz von kognitiver und kulturwissenschaftlicher Semantik am Beispiel von Begriffsgeschichte, Diskursanalyse und FrameSemantik. In: Kämper, H. / Eichinger, L. (Hrsg.). Sprache - Kognition - Kultur. Sprache zwischen mentaler Struktur und kultureller Prägung. (= Jahrbuch 2007 des Instituts für deutsche Sprache) Berlin/New York: de Gruyter, 73-114. <http://www.phil-fak.uni-duesseldorf. de/fileadmin/Redaktion/Institute/Germanistik/AbteilungI/Busse/Texte/Busse-2008-02ur.pdf>.

Busse, D. 2008d. Semantische Rahmenanalyse als Methode der Juristischen Semantik. Das verstehensrelevante Wissen als Gegenstand semantischer Analyse. In: Christensen, R. / Pieroth, B. (Hrsg.). Rechtstheorie in rechtspraktischer Absicht. Freundesgabe zum 70. Geburtstag von Friedrich Müller. (=Chriften zur Rechtssprache 235) Berlin: Duncker \& Humblot, 35-55. <http://www.phil-fak.uni-duesseldorf.de/fileadmin/Redaktion/Institute/Germanistik/ AbteilungI/Busse/Texte/Busse-2008-01-ur.pdf>.

Busse, D. 2007a. Diskurslinguistik als Kontextualisierung - Sprachwissenschaftliche Überlegungen zur Analyse gesellschaftlichen Wissens. In: Warnke, I. (Hrsg.). Diskurslinguistik nach Foucault. Berlin: de Gruyter, 81-105. <http://www.phil-fak.uni-duesseldorf.de/filea dmin/Redaktion/Institute/Germanistik/AbteilungI/Busse/Texte/Busse-2007-01-ur.pdf>. 
Busse, D. 2007b. Linguistische Epistemologie in der Weltgesellschaft. Theoretische und methodische Anmerkungen zur semantischen Analyse "globalisierter" Diskurse. In: Wengeler, M. / Ziem, A. (Hrsg.). „Globalisierung“ als diskurssemantische Grundfigur? Bedeutungsdimensionen - Argumentationsmuster - diskursive Strategien. (= Aptum - Zeitschrift für Sprachkritik und Sprachkultur, 3. Jahrgang, 2007, H. 02, 105-121. <http://www.phil-fak.uni-duesseldorf. de/fileadmin/Redaktion/Institute/Germanistik/AbteilungI/Busse/Texte/Busse-2007-03ur.pdf>.

Busse, D. 2005. Architekturen des Wissens - Zum Verhältnis von Semantik und Epistemologie. In: Müller E. (Hrsg.). Begriffsgeschichte im Umbruch. (= Archiv für Begriffsgeschichte, Sonderheft 2004) Berlin: Felix Meiner, 2005, 43-57. <http://www.phil-fak.uni-duesseldorf.de/ fileadmin/Redaktion/Institute/Germanistik/AbteilungI/Busse/Texte/Busse-2005-03.pdf>.

Busse, D. 1992. Textinterpretation: Sprachtheoretische Grundlagen einer explikativen Semantik. Opladen: Westdeutscher Verlag.

Dietze, J. 1994. Texterschließung: Lexikalische Semantik und Wissensrepräsentation. München: de Gruyter.

Devitt, M. 2012. Semantic Epistemology: Response to Machery. In: Theoria, 74, 229-233.

Engelkamp, J. 1973. Semantische Struktur und die Verarbeitung von Sätzen. Bern: Huber.

Engelmann, J. B. / Hein, G. 2013. Centextual and Social Influences on Valuation and Choice. In: Pammi, C. V. S. / Srinivasan (Hrsg.). Decision Making. Neuronal and Behavioural Approaches. Amsterdam: Elsevier, 215-238.

Fillmore, Ch. J. 1982. Frame Semantics. The Linguistics Society of Korea (Hrsg.) Linguistics in the Morning Calm. Seul: Harnishin Publishing, 111-137.

Frisk, H. 1954-1972. Griechisches Etymologisches Wörterbuch. Heidelberg, Bd. 1., 1-XXX, 1-938 [aко] 1960; Bd. 2., 1-1154 [к $\rho-\omega]$ 1970; Bd. 3., 1-312 [Nachträge, Wortregister, Corrigenda] (1972) <http://ieed.ullet.net/friskL.html>.

Geckeler, H. 1971. Zur Wortfelddiskussion : Untersuchungen zur Gliederung des Wortfeldes, alt jung - neu' im heutigen Französisch. München: Fink.

Grabowski, J. / Harras, G. / Herrmann, Th. (Hrsg.) 1996. Bedeutung, Konzepte, Bedeutungskonzepte: Theorie und Anwendung in Linguistik und Psychologie. Opladen: Westdeutscher Verlag.

Grucza, F. 2012a. Kulturologia antropocentryczna a kulturoznawstwo. In: Grzywka, K. / Filipowicz, M. / Godlewicz-Adamiec, J. / Jagłowska, A. et al. (Hrsg.). Kultura - Literatura Jezyk / Kultur - Literatur - Sprache, Bd. 1., Warszawa: Instytut Germanistyki UW, 79-101.

Grucza, F. 2012b. Zum Gegenstand und zu den Aufgaben der anthropozentrischen Linguistik, Kultlturologie und Kommunikologie sowie zur gegenseitigen Vernetzung dieser Erkenntnisbereiche. In: Kwartalnik Neofilologiczny, 59, 3, 287-344.

Grucza, F. 2010. Zum ontologischen Status menschlicher Sprachen, zu ihren Funktionen, den Aufgaben der Sprachwissenschaft und des Sprachunterrichts. In: Kwartalnik Neofilologiczny, 3, 257-274.

Grucza, F. 2000. Kultur aus der Sicht der Angewandten Linguistik. In: Schlosser, H. G., (Hrsg.). Sprache und Kultur. Frankfurt/M.: Peter Lang (Forum Angewandte Linguistik. 38), 17-30.

Grucza, F. 1994. Języki specjalistyczne - ich rozumienie i znaczenie. In: ders. / Kozłowska, Z. (Hrsg.). Jezyki specjalistyczne. Materiaty XVIII Sympozium ILS UW (Warszawa, 9-11 stycznia 1992). Warszawa: Instytut Lingwistyki Stosowanej UW, 7-27.

Grucza, F. 1992. Kulturowe determinanty języka oraz kompetencji językowej. In: ders. (Hrsg.). Jezyk, kultura - kompetencja jezykowa. Materiaty XIII sympozjum Instytutu Lingwistyki Stosowanej UW. Zaborów: 5-8 listopada 1987. Warszawa: Instytut Lingwistyki Stosowanej UW, 9-70. 
Grucza, F. 1991. Terminologia. Jej przedmiot, status i znaczenie. In: ders. (Hrsg.), Teoretyczne podstawy terminologii. Wrocław, Warszawa, Kraków: Zakład Narodowy Ossolińskich (Seria Komitetu Terminologii. Problemy Treminologii. 1.), 11-44.

Grucza, F. 1988. Zum Begriff der Sprachkompetenz, Kommunikationskompetenz und Kulturkompetenz. In: Honsza, N., Roloff, H.-G. (Hrsg.). Daß eine Nation die ander verstehen möge: Festschrift für Marian Szyrocki zu seinem 60. Geburtstag. Amsterdam: Rodopi (Chloe Amsterdam. 7.), 309-332.

Grucza, F. 1983. Zagadnienia metalingwistyki. Lingwistyka - jej przedmiot, lingwistyka stosowana. Warszawa: PWN.

Grucza, S. 2013. Język a poznanie - kilka uwag na temat rozważania ich wzajemnych relacji. In: ders. / Marchwiński, A. / Płużyczka, M. (Hrsg.). Translatoryka. Koncepcje - Modele Analizy. Księga jubileuszowa ofiarowana Profesor Barbarze Z. Kielar z okazji 80. rocznicy urodzin. Warszawa: WUW, 54-67.

Grucza, S. 2012. Fachspachenlinguistik. Frankfurt/M.: Peter Lang (Warschauer Studien zur Germanistik und Angewandter Linguistik 1.)

Grucza, S. 2010. Główne tezy antropocentrycznej teorii jezyków. In: Lingwistyka Stosowana / Applied Linguistics, 2, 41-68.

Grucza, S. 2008. Lingwistyka języków specjalistycznych. Warszawa: Euroedukacja (Języki - Kultury - Teksty - Wiedza).

Grzegorczykowa, R. 2001. Wprowadzenie do semantyki jezzykoznawczej. Warszawa: PWN.

Helbig, H. 2008. Wissensverarbeitung und die Semantik der Natürlichen Sprachen. Berlin, Heidelberg: Springer.

Katz, J. J. / Foder J. A. 1964. The Structure of Semantic Theory. Engelwood Cliffs: Prentice Hall.

Köbler, G. 1995. Deutsches Etymologisches Wörterbuch. Tübingen: UTB <http://www.koebler gerhard.de/derwbhin.html>.

Kleiber, G. 2003. Semantyka prototypu. Kategorie i znaczenia leksykalne. Kraków: Universitas.

Landels, J. G. 1999. Ancient in Ancient Greece and Rome. London: Routledge.

Landels, J. G. 2003. Muzyka starożytnej Grecji i Rzymu. Kraków: Homini.

Linz, E. 2002. Indiskrete Semantik. Kognitive Linguistik und neurowissenschaftliche Theoriebildung. München: Fink.

Loppe, T. 2010. Bedeutungswissen und Wortgebrauch. Entwurf einer Semantik im Anschluss am Wittgenstein und Putnam. Tübingen: Narr.

Löbach, B. 1999. Semantikerwerb: ein Beitrag zu einer empiristisch-naturalistischen Bedeutungstheorie. Tübingen: Niemeyer.

Löbner, S. 2003. Semantik. Eine Einführung. Berlin / New York: de Gruyter.

Lutzeier, P. R. 1985. Linguistische Semantik. Stuttgart: Metzler.

Lyons, J. 1977. Semantics. Vol. 1/2. London, NY, Melbourne: Cambridge University Press.

Mills, S. 2007. Der Diskurs: Begriff, Theorie, Praxis. München: UTB.

Mangasser-Wahl, M. 2000. Von der Prototypentheorie zur empirischen Semantik. Dargestellt am Beispiel von Frauenkategorisierungen. Frankfurt/M., et al.: Peter Lang.

Metzeltin, M. 2007. Theoretische und angewandte Semantik. Vom Begriff zum Text. Wien: Praesens Verlag.

Montague, P. R. / Berns G. S. 2002. Neural Economics and the Biological Substrates of Valuation. In: Neuron 36(2), 265-284.

Northoff, G. 2014. Unlocking the Brain. Vol. 1. Coding. Oxford: Oxford University Press.

Pawłowski, G. 2015a. Kognitiv und / oder epistemisch. Auf dem Weg zur epistemologischen Semantik. In: Glottodidactica, 42, 1/2015, 65-81. 
Pawłowski, G. 2015b. ,auleta' und es bleibt alles beim Neuen!? Zur Konstitution der polnischen Fachneologismen im Bereich der altgriechischen Musik. In: Zeitschrift des Verbandes Polnischer Germanisten, (4)1, 45-63.

Pawłowski, G. 2015c. Translatorische Implikationen bei der Konstitution von polnischen Fachneologismen im Fachgebiet, Byzantinische Musik und Hymnografie'. In: Weigt, Z. / Kaczmarek, D. / Makowski, J. / Michon', M. (Hg.): Felder der Sprache - Felder der Forschung. Lodzer Germanistikbeiträge III, Bd. 2., Łódź: Verlag der Universität Łódź, 87-102.

Pawłowski, G. 2014. „Eh, ich sing ja nicht dazu, um gehört zu werden“. Ein Beitrag zur Bedeutungskonstitution idiolektaler Ausdrücke am Beispiel gesungener Texte aus dem polnischen West-Roztocze. In: Żebrowska, E. / Steinhoff, D. / Jaworska, M. (Hrsg.). Materialität und Medialität der sprachlichen Kommunikation. Frankfurt am Main, et. al. (Linguistik International. Akten des 47. Linguistischen Kolloquiums in Olsztyn), 265-287.

Pawłowski, G. 2013a. Zur linguistischen Typologie von Konnotationen aus der Sicht der epistemologisch profilierten Semantik. In: Kwartalnik Neofilologiczny, 60, 3, 291-337. <http:// kn.czasopisma.pan.pl>.

Pawłowski, G. 2013b. Bedeutungskonstitution: Zu epistemischen Formanten der idiolektalen Bedeutung. In: Bartoszewicz, I., / Szczęk, J. / Tworek, A. (Hrsg.). Linguistische Treffen in Wrocław 9. Im Anfang war das Wort II. Wrocław, Dresden, 201-211.

Pawłowski, G. 2012. Zum Gegenstand der linguistischen Semantik. In: ders. / OlpińskaSzkiełko, M. / Bonacchi, S. (Hrsg.). Mensch - Sprachen - Kulturen, Warszawa, 249-263 und Zeitschrift des Verbandes Polnischer Germanisten, H. 3, 249-263 <http://www.ejournals.eu/ ZVPG/Tom-2012/Zeszyt-3-(2012)/>.

Pawłowski, G. 2011. Bedeutungstransfer - möglich oder unmöglich. Eine linguistische Analyse wissenschaftlicher Texte. In: Grucza, F /, Zimniak, Z. / Pawłowski, G. (Hrsg.). Die deutsche Sprache, Kultur und Literatur in polnisch-deutscher Interaktion. Warszawa: Euroedukacja, 53-67.

Pawłowski, G. 2010. Heißt konnotativ peripher? Ein Beitrag zur anthropozentrischen Sprachentheorie dargestellt an einer kontrastiven Studie zu den idiolektalen Konnotationen der Lexeme Kirche und kościół. In: Małgorzewicz, A. (Hrsg.). Translation: Theorie - Praxis Didaktik. Wrocław, Dresden: Neisse, 213-226.

Pawłowski, G. 2009. Freiheit - Lexemgrenzen aus semantischer Sicht. In: Babenko, N. S. / Bakshi, N. A. (Hrsg.). Russische Germanistik. Jahrbuch des Russischen Germanistenverbandes. Moskau 6: Institut für deutsch-russische Literatur-und Kulturbeziehungen, 381-394.

Polenz, P. v. 1988. Deutsche Satzsemantik. Grundbegriffe des Zwischen-den-Zeilen-Lesens. 2., durchges. Aufl. Berlin, New York: de Gruyter.

Reis, M. 1980. Grundbegriffe der Semantik. Köln: Arbeitspapier Uni Köln.

Radeiski, B. 2011. Seuchen, Ängste und Diskurse: Massenkommunikation als diskursives Rollenspiel. Berlin, New York: de Gruyter.

Sachs, C. 1981/1988. Muzyka w świecie starożytnym. Übers. v. Z. Chechlińska. Warszawa: Polskie Wydawnictwo Muzyczne.

Schemann, H. 2002. Idiomatik und Anthropologie. "Bild“ und "Bedeutung" in linguistischer, sprachgenetischer und philosophischer Perspektive. Hildesheim / Zürich / New York: Olms.

Schwarz, M. 1992. Kognitive Semantiktheorie und neurophysiologische Realität. Repräsentationale und prozedurale Aspekte der semantischen Kompetenz. Tübingen: Niemeyer.

Schroll-Machl, S. / Wiskoski, K. 1999. Typisierte polnisch-deutsche Kulturunterschiede: Welche business-relevante Werte, Grundhaltungen und Grundeinstellungen sind zwischen Polen und Deutschen verschieden?. In: Rösch, O. (Hrsg.). (1999). Interkulturelle Kommuni- 
kation mit polnischen Partnern in Wirtschaft und Wissenschaft. Berlin: News \& Media Marcus v. Amsberg, 55-64.

Tokarski, R. 2004. Semantyka barw we współczesnej polszczyźnie. Lublin: UMCS.

Tokarski, R. / Pajdzińska, A. 2001. Semantyka tekstu artystycznego. Lublin: UMCS.

The New Grove Dictionary of Music and Musicians (Hrsg.) Stanley S. / Johan T., Bd. 2. Oxford et. al.: Aristoxenus to Bax.

Wierzbicka, A. 2007. Stowa klucze. Różne jezzyki - różne kultury. Warszawa: WUW.

Wierzbicka, A. 2006. Semantyka. Jednostki elementarne i uniwersalne. Lublin: UMCS.

West, M. L. 1992. Ancient Greek Music. New York: Oxford University Press

West, M. L. 2003. Muzyka starożytnej Grecji. Kraków: Homini.

Wellesz, E. 1961. A History of Byzantine Music and Hymnography. New York: At the Clarendon Press.

Wellesz, E. 2006. Historia muzyki i hymnografii bizantyjskiej. Kraków: Homini.

Wunder, A. 2008. Die Semantik der genetischen Information. Eine Untersuchung ausgewählter Texte. Saarbrücken: Vdm

Ziem, A. 2008. Frame und sprachliches Wissen. Kognitive Aspekte der semantischen Kompetenz. Berlin / New York: de Gruyter.

Ziem, A. 2005. Begriffswissen. Ein linguistischer Beitrag zur sprachlichen Bedeutungskonstruktion in literarischen Texten. In: Roussel, M. / Wirtz, M. / Wunderlich, A. (Hrsg.). Eingrenzen und Überschreiten. Verfahren in der Moderneforschung. Würzburg: Königshausen \& Neumann, 272-285. 
